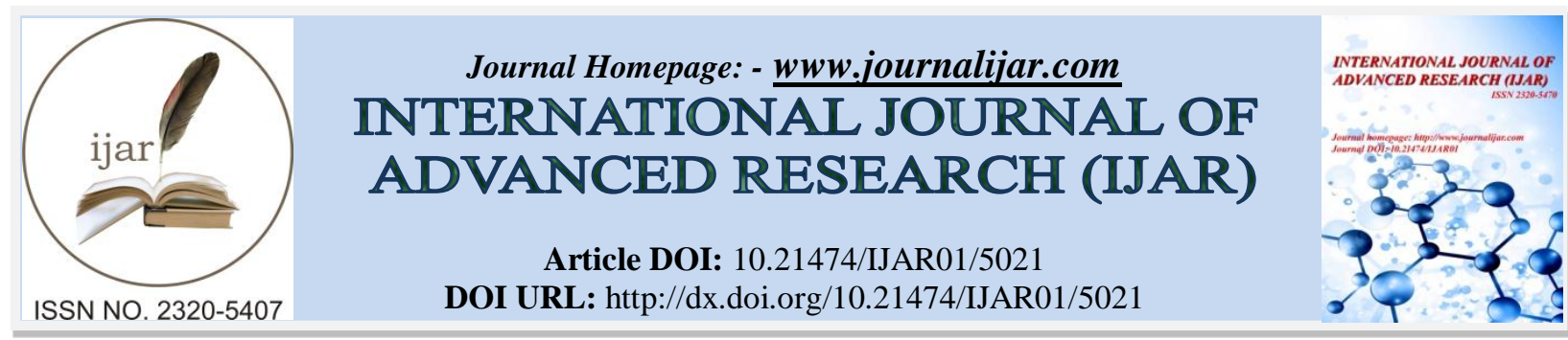

RESEARCH ARTICLE

\title{
SYNTHESIS, CHARACTERIZATION AND PHYSICO-CHEMICAL PROPERTIES OF COPOLYESTERS ONTAINING $s$-TRIAZINE RING IN THE MAIN CHAIN.
}

\author{
Rahul B. Tailor, Premlata H. Patel, Mohsin A. Belim, Savan V. Patel, Sejal M. Patel, Hushen U. Hajiyani, \\ Paresh S. Patel and *Keshav C. Patel. \\ Department of Chemistry, Veer Narmad South Gujarat University, Udhna-Magdalla Road, Surat-395 007, Gujarat,
} India.

\section{Manuscript Info}

Manuscript History

Received: 28 May 2017

Final Accepted: 30 June 2017

Published: July 2017

Key words:-

Copolyesters, Density, Physico-

chemical, Thermal stability, Viscosity

\begin{abstract}
A series of copolyesters has been synthesized by polycondesation of 2( $N$-piperidino)-4,6-bis (2-napthoxy, 6-carbonyl chloride)-s-triazine [PNCCT] with various mixture of diols, such as $[\mathrm{Hq}+\mathrm{BPA}]$, $[\mathrm{C}+$ $\mathrm{BPA}],[\mathrm{R}+\mathrm{BPA}],[\mathrm{C}+\mathrm{BPC}],[\mathrm{R}+\mathrm{BPC}],[\mathrm{BPC}+\mathrm{BPS}],[\mathrm{Hq}+\mathrm{Ph}]$, $[\mathrm{Hq}+\mathrm{C}],[\mathrm{Hq}+\mathrm{BPS}]$ and $[\mathrm{Hq}+\mathrm{Eg}]$. All the synthesized copolyesters were characterized by solubility, density, viscosity, IR, ${ }^{1} \mathrm{H}$ NMR and TGA spectra. Modification and introduction of bulky pendent group in monomer structure is increases the thermal stability and solubility of the polymers. The density of all the copolyesters varies from 1.204 to $1.165 \mathrm{~g} / \mathrm{cm}^{3}$. The intrinsic viscosity, reduced viscosity and inherent viscosity were determined by using Huggins \& Kraemer method. The activation energy was calculated by Broido and Horowitz \& Metzger method for PE 8 (II) and PE 9 (II) were found 21.30 and 18.69 $\mathrm{kcal} / \mathrm{mol}$ respectively. The synthesized polymers are found to stable more than $300{ }^{\circ} \mathrm{C}$ temperature so it can be used for high temperature applications.
\end{abstract}

Copy Right, IJAR, 2017,. All rights reserved.

\section{Introduction:-}

Today's requirement of novel polymers with improved property has been expanding with time and it can be said to be endless. Polymeric materials have potential use as rubbers, resins, plastics, composites, adhesives, laminates and coatings. The polymers science beginning and headway in the second and third decade of the twentieth century. Though introduced very late, polymers occupy a major place and pivotal position in our materials map today. In application prospects, performance and versatility in property that can hardly be occupied by any other kind of materials [1-4]. A literature survey reveals that the polymers based on $s$-triazine moiety and their derivatives have been extensively used since last few years. Recently most researchers working for the synthesis of high-performance polymers with high thermal stability and heat resistance with good mechanical properties, as they are required to use as engineering plastic materials. Now a day the copolyesters are largely used in various fields to achieve high thermal stability and good mechanicals property [5-8]. Polymers containing $s$-triazine ring in the main chain have excellent heat resistance and several are commercially available as high-performance engineering plastic materials. Hence, it was thought interesting to synthesize and characterize some copolyesters based on $s$-triazine [9-11]. In present investigation, we have synthesized and characterized copolyesters from diacid chloride of 2 -( $N$-piperidino)4,6-bis(2-napthoxy,6-carboxy)triazine and various equimolar mixtures of aromatic diols. 


\section{Materials:-}

Fresh double-distilled water was used for the preparation of solutions. Solvents, like benzene, acetone, N,Ndimethyl formamide, carbon tetrachloride, methanol, ethanol, dimethyl sulphoxide, nitrobenzene, pyridine and hexane were all laboratory reagents, obtained from Merck and used as received. Cyanuric chloride (Fulka) was purified by repeated crystallization from pure benzene (m.p. $\left.146^{\circ} \mathrm{C}\right)$.

The resin grade bisphenol-A received form Atul Ltd. was repeatedly crystallized from 50\% aqueous acetic acid. The crystals of bisphenol-A were filtered, thoroughly washed with water and dried. It was purified by recrystallization from benzene (m.p. $156{ }^{\circ} \mathrm{C}$ ). Bisphenol-C was synthesized following a published procedure [12] and was crystallized from benzene (m.p. $187^{\circ} \mathrm{C}$ ). Phenolphthalein (BDH, m.p. $262^{\circ} \mathrm{C}$ ) was purified by crystallization from ethanol. Resorcinol, catechol and hydroquinone (Sisco lab) were purified by recrystallization from rectified spirit and 1,5-dihydroxy naphthalene (Sisco lab) was purified by recrystallization from aqueous alcohol. Ethylene glycol of better purity (Merck) was used as received.

\section{Experimental:-}

\section{Step I: Synthesis of 2-( $N$-piperidino)-4, 6-dichloro-s-triazine [PDCT]:-}

A solution of cyanuric chloride (18.44 g, 0.1 mole) in $60 \mathrm{ml}$ acetone was added with stirring in a cold solution $\left(0-5{ }^{\circ} \mathrm{C}\right)$ of sodium bicarbonate $(10.6 \mathrm{~g})$ in $100 \mathrm{ml}$ distilled water, in three necked $250 \mathrm{ml}$ flask equipped with a mechanical stirrer. This results in the formation slurry of cyanuric chloride. A solution of piperidine $(8.51 \mathrm{~g}, 0.1$ mole) in $10 \mathrm{ml}$ acetone was added to the cold slurry of cyanuric chloride with continuous stirring. After addition was completed, the reaction mixture was stirred for $2 \mathrm{hr}$ at $0-5{ }^{\circ} \mathrm{C}$. The light yellow colored product was filtered and recrystallized from ethanol and dried in a vacuum desiccator (yield $81 \%$, m.p. $90{ }^{\circ} \mathrm{C}$ ) [13-14].

\section{Scheme 1:-}

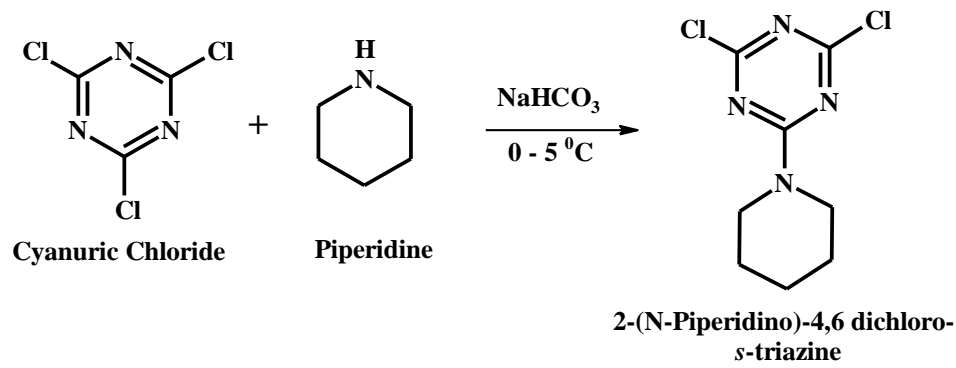

Step II: Synthesis of 2-( $N$-piperidino)-4, 6-bis (2-napthoxy, 6-carboxy) triazine [PNCT]:-

A solution of 6-hydroxy-2-naphthoic acid (37.6g, 0.2 mole) in $\mathrm{NaOH}(16.0 \mathrm{~g}, 0.4 \mathrm{~mole})$ and $80 \mathrm{ml}$ double distilled water was added slowly to 2-( $N$-piperidino)-4,6-dichloro-s-triazine [PDCT] (23.4 g, $0.1 \mathrm{~mole})$ in $40 \mathrm{ml}$ of acetone at room temperature with constant stirring. Reaction was continued for $4 \mathrm{hr}$ first $2 \mathrm{hr}$ at room temperature and then another $2 \mathrm{hr}$ at $80^{\circ} \mathrm{C}$. At the end of the reaction, the white solid separated from the reaction mixture was filtered and washed several times with hot water and dried in vacuum at $100{ }^{\circ} \mathrm{C}$. The product was recrystallized from acetone (yield was $80 \%$, m.p. $234{ }^{\circ} \mathrm{C}$ ) [15-16].

\section{Scheme 2:}

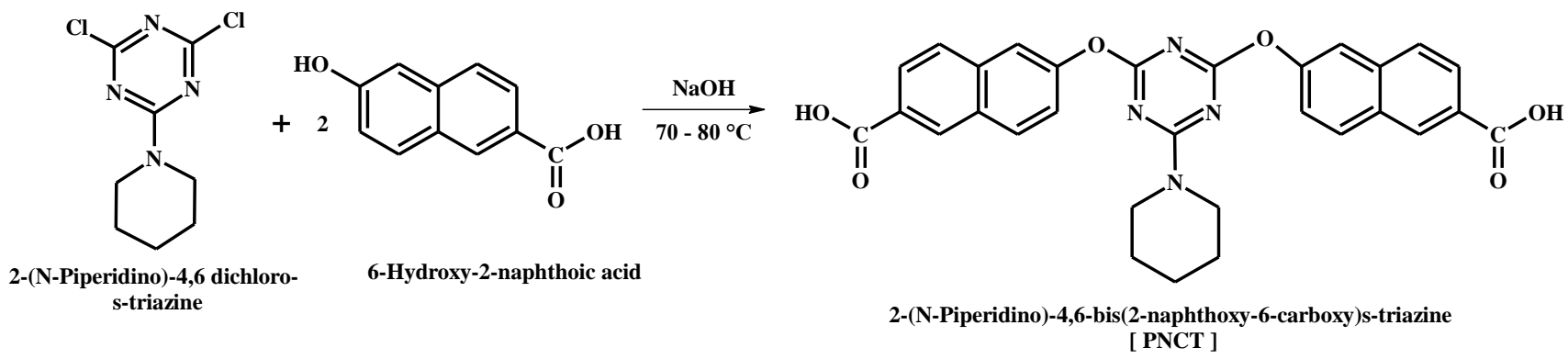


Step III: Synthesis of 2-( $N$-piperidino)-4, 6-bis (2-napthoxy, 6-carbonyl chloride)-s-triazine [PNCCT]:-

Place 2-( $N$-piperidino)-4,6-bis(2-napthoxy,6-carboxy)-s-triazine [PNCT] (5.36 g, 0.01 mole) in a dry round bottom flask. Thionyl chloride $(11.9 \mathrm{~g}, 0.1 \mathrm{~mole})$ was added at the top of the condenser and the reaction mixture was refluxed at $78{ }^{\circ} \mathrm{C}$ for $2 \mathrm{hr}$. At the end of the reaction, the excess of thionyl chloride was distilled off and dry product was recovered. The yield recrystallized from dimethyl formamide (yield about $78 \%$ and m.p. $205{ }^{\circ} \mathrm{C}$ ) [17-18].

Scheme 3:<smiles>O=C(O)c1ccc2cc(Oc3nc(Oc4ccc5cc(C(=O)O)ccc5c4)nc(N4CCCCC4)n3)ccc2c1</smiles>

\section{Step IV: Synthesis of copolyesters from PNCCT and various mixture of diols:-}

The copolyesters were synthesized by polycondensation of 2-( $N$-piperidino)-4,6-bis(2-napthoxy,6-carbonylchloride)s-triazine $[\mathrm{PNCCT}]$ and various mixture of diols, such as $[\mathrm{Hq}+\mathrm{BPA}],[\mathrm{C}+\mathrm{BPA}],[\mathrm{R}+\mathrm{BPA}],[\mathrm{C}+\mathrm{BPC}],[\mathrm{R}+$ $\mathrm{BPC}],[\mathrm{BPC}+\mathrm{BPS}],[\mathrm{Hq}+\mathrm{Ph}],[\mathrm{Hq}+\mathrm{C}],[\mathrm{Hq}+\mathrm{BPS}]$ and $[\mathrm{Hq}+\mathrm{Eg}][19]$.

\section{Scheme 4:}

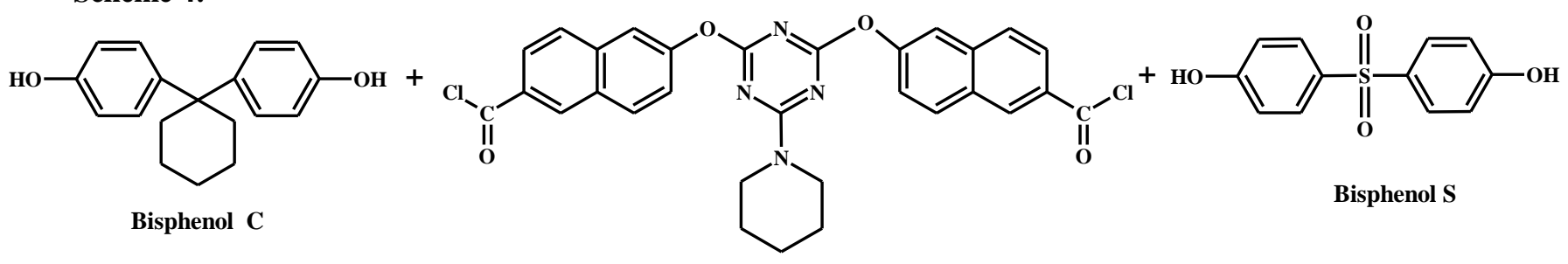
Diacid chloried
2-(N-Piperidino)-4,6-bis(2-naphthoxy-6-carboxy)s-triazine [ PNCT ]

\begin{tabular}{c|} 
DMF \\
Cetrimide \\
$165^{\circ} \mathrm{C}$
\end{tabular}<smiles></smiles>

PE 6 (II) PE (BPC + BPS)

\section{Measurements:-}

FTIR spectra of all the polyesters were recorded on Perkin Elmer FTIR Paragon 1000 using KBr pellet technique. The NMR spectra were recorded on a Perkin-Elmer Model-32 ${ }^{1} \mathrm{H}$ NMR spectrometer (400 MHz). Density of the polymers has been determined pyknometrically at $25{ }^{\circ} \mathrm{C}$ using the suspension method. Solution viscosity measurements have been carried out for the homopolyesters. Reduced viscosities of polyesters were determined for $1 \mathrm{gm} / \mathrm{dl}$ solution in DMF using an Ubbelohde suspended level viscometer. Intrinsic viscosity was obtained from the 
plot of reduced viscosity vs. concentration and inherent viscosity vs. concentration according to Huggins [20] and Kraemer [21] equations. Thermogravimetric analysis of some selected homopolyesters has been carried out on the thermobalance Metter TA-4000 system with constant heating rate of $10{ }^{\circ} \mathrm{C} / \mathrm{min}$ in the temperature range from ambient to $900{ }^{\circ} \mathrm{C}$. All TGA have been analyzed as per the graphical methods proposed by Broido [22] and Horowitz \& Metzger [23].

\section{Result and Discussion:-}

Various copolyesters were synthesized from PDCT and Mixture of different diols. The synthesized copolyesters have been evaluated for various physicochemical properties such as yield, color, solubility, density, viscosity, temperature characteristics, activation energy of thermal decomposition, IR and ${ }^{1} \mathrm{H}$ NMR spectroscopy.

\section{Infrared Spectra:-}

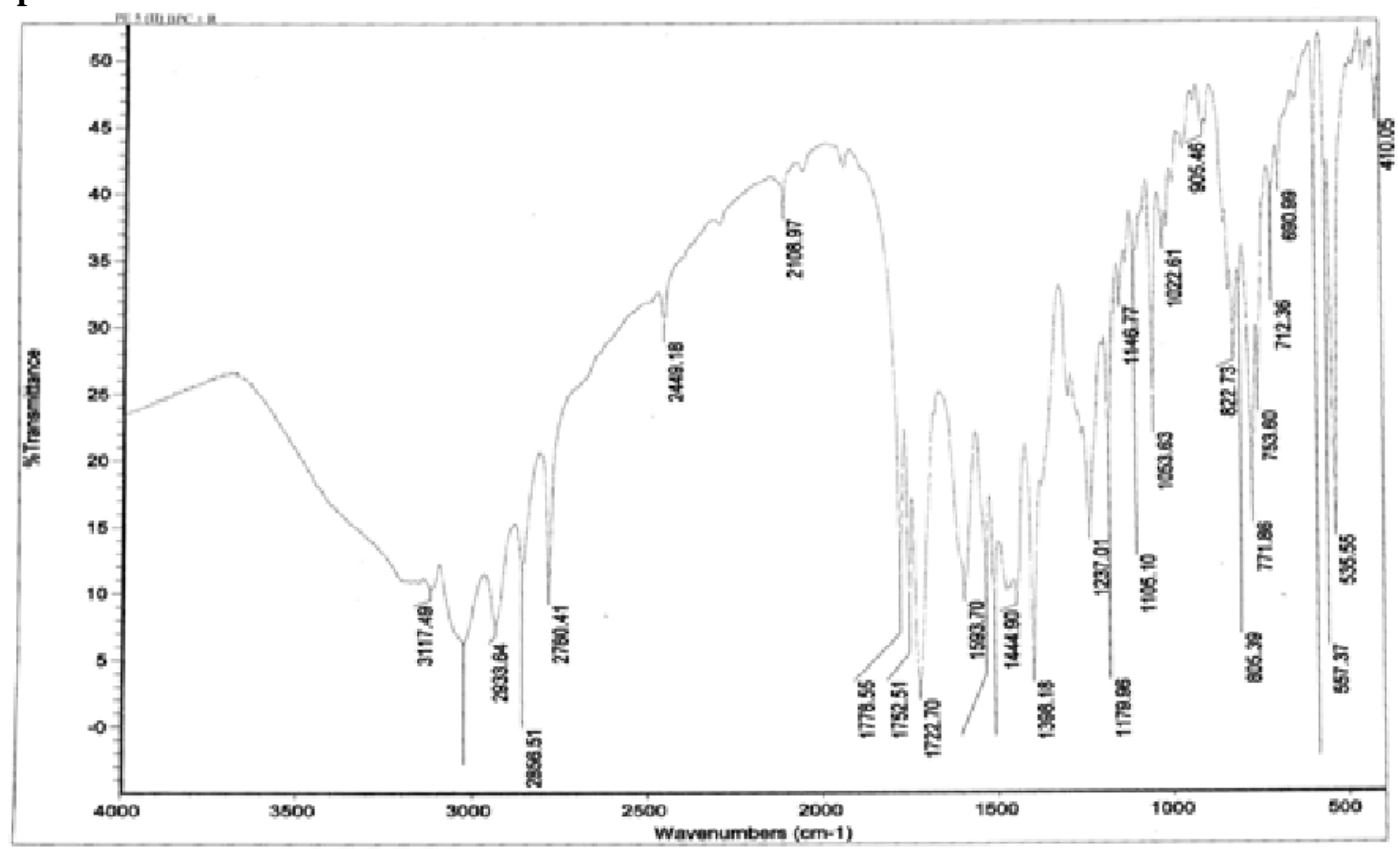

Figure_1 IR spectrum of PE 5 (II)

The IR spectral of PE 5 (II) showed deformation vibrational band of cyclohexane link at $557 \mathrm{~cm}^{-1}$, m-substitution on phenyl ring at $712 \mathrm{~cm}^{-1}, \mathrm{C}-\mathrm{H}$ bending vibration of aromatic ring at 753-557 $\mathrm{cm}^{-1}$, out of plane vibration of $s$-triazine ring at $805 \mathrm{~cm}^{-1}, \mathrm{C}-\mathrm{H}$ bending vibration of aromatic ring at $905 \mathrm{~cm}^{-1}$, Vibration of aryl ether linkage symmetric at $1053 \mathrm{~cm}^{-1}, \mathrm{C}-\mathrm{C}(=\mathrm{O})$-O ester stretching symmetric at $1179 \mathrm{~cm}^{-1}$, aromatic $\mathrm{C}-\mathrm{N}$ stretching at $1337 \mathrm{~cm}^{-1}$, $\mathrm{C}-\mathrm{H}$ bending vibration of methyl group at $1389 \mathrm{~cm}^{-1}$, in plane vibration of $s$-triazine ring at $1444-1494 \mathrm{~cm}^{-1}$, C-C $(=\mathrm{O})-\mathrm{O}$ ester stretching vibration at $1752 \mathrm{~cm}^{-1}, \mathrm{C}-\mathrm{H}$ stretching vibration of methyl group at $2780 \mathrm{~cm}^{-1}$, $\mathrm{C}-\mathrm{H}$ stretching vibration of methylene group at $3020 \mathrm{~cm}^{-1}$. 


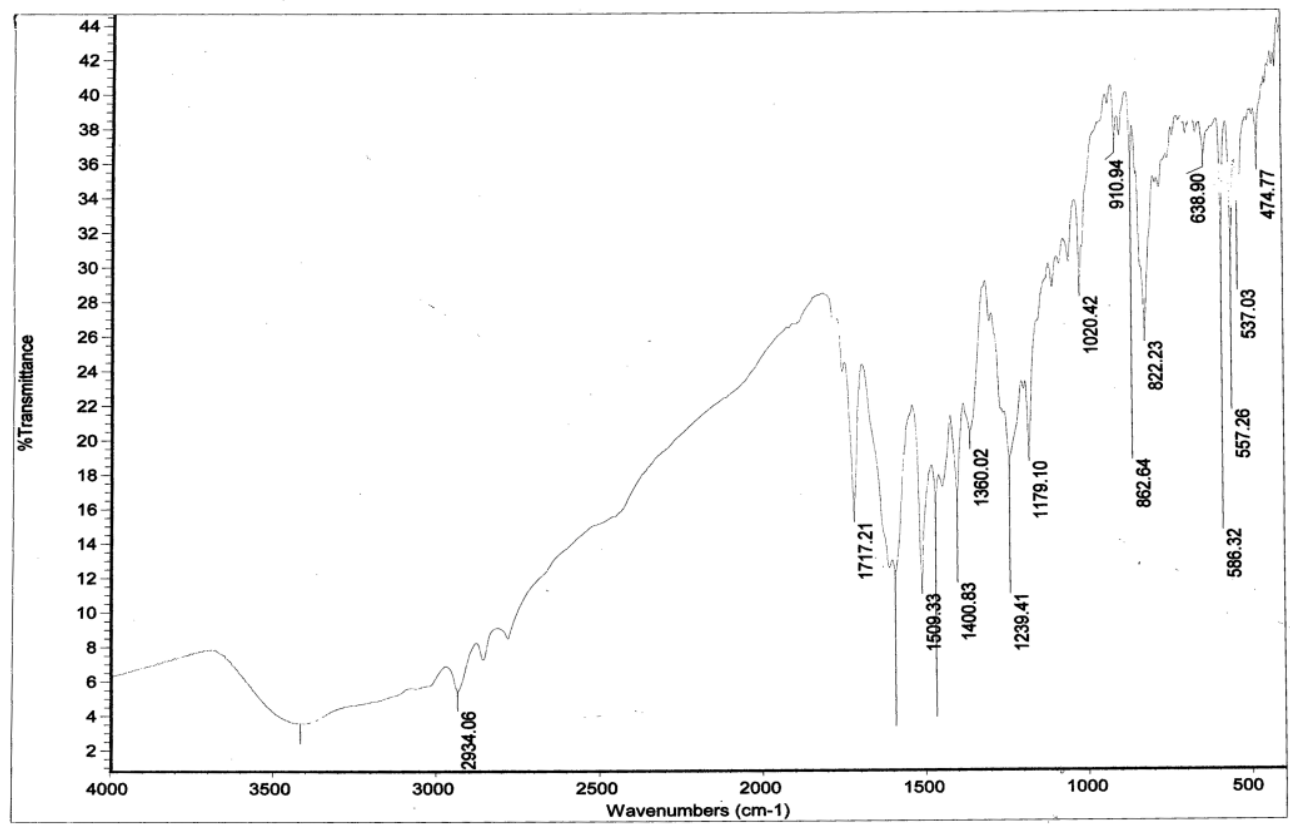

Figure_2 IR spectrum of PE 6 (II)

IR spectral data of PE 6 (II) showed out of plane vibration of $s$-trizaine ring at $822 \mathrm{~cm}^{-1}, \mathrm{SO}_{2}$ stretching vibration of sulphone group at $1179 \mathrm{~cm}^{-1}, \mathrm{C}-\mathrm{H}$ bending vibration of aromatic ring at $910 \mathrm{~cm}^{-1}$, vibration of aryl ether linkage symmetric at $1020 \mathrm{~cm}^{-1}, \mathrm{C}-\mathrm{C}(=\mathrm{O})-\mathrm{O}$ ester stretching symmetric at $1179 \mathrm{~cm}^{-1}$, aromatic $\mathrm{C}-\mathrm{N}$ stretching vibration $1360 \mathrm{~cm}^{-1}, \mathrm{C}-\mathrm{H}$ bending vibration of methyl group at $1400 \mathrm{~cm}^{-1}$, in plane vibration of $s$-triazine ring at $1509 \mathrm{~cm}^{-1}$, $\mathrm{C}-\mathrm{C}(=\mathrm{O})-\mathrm{O}$ stretching vibration of $1717 \mathrm{~cm}^{-1}, \mathrm{C}-\mathrm{H}$ stretching vibration of methylene group at $2934 \mathrm{~cm}^{-1}$.

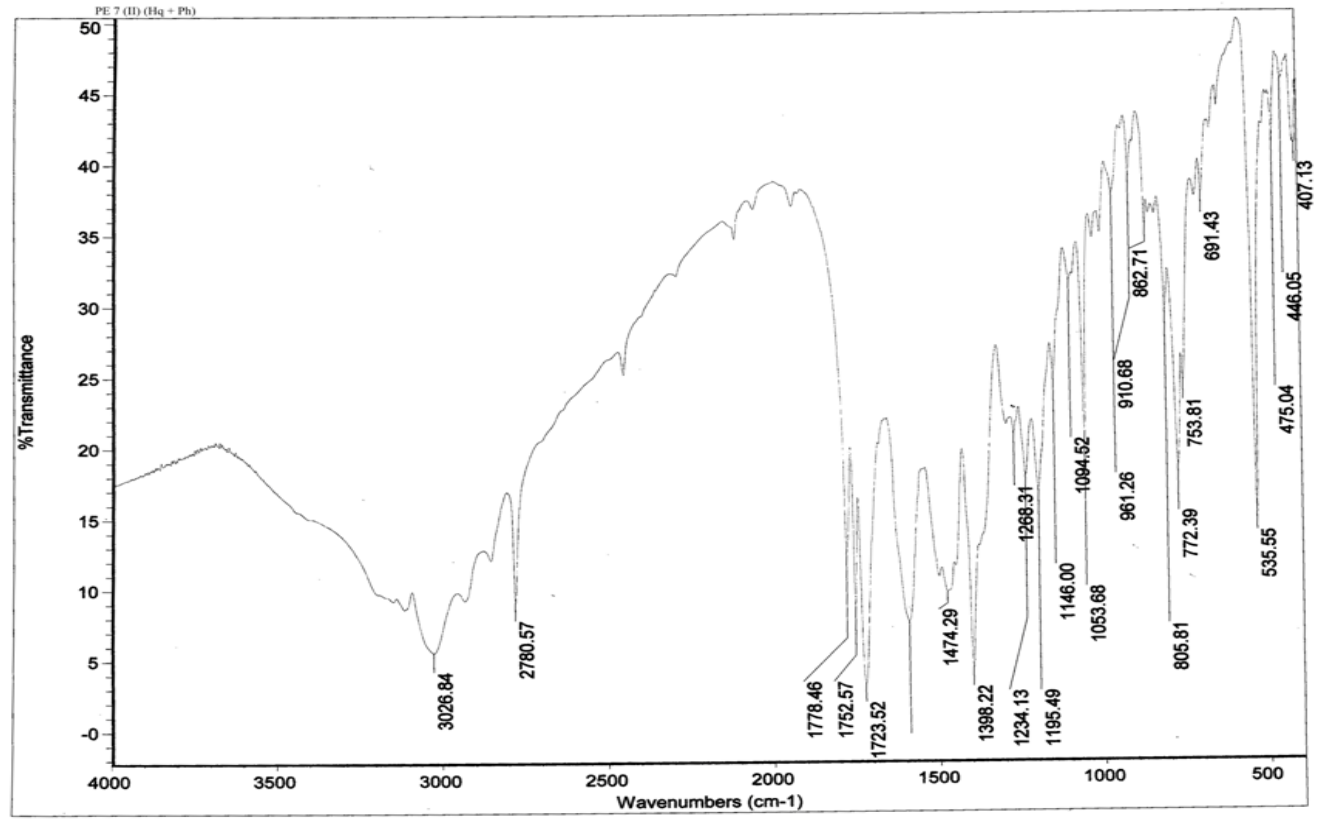

Figure_3 IR spectrum of PE 7 (II)

IR spectral data of PE 7 (II) in out of plane bending vibration of C-H bond of aromatic ring at $753-772 \mathrm{~cm}^{-1}$, out of plane vibration of $s$-triazine ring at $805 \mathrm{~cm}^{-1}, \mathrm{C}-\mathrm{H}$ bending vibration of aromatic ring at $862 \mathrm{~cm}^{-1}$, vibration of aryl ether linkage symmetric at $1053 \mathrm{~cm}^{-1}$, C-C (=O)-O ester stretching symmetric at $1146 \mathrm{~cm}^{-1}$, Vibration of aryl ether linkage Asymmetric at $1268 \mathrm{~cm}^{-1}$, C-H bending vibration of methyl group at $1396 \mathrm{~cm}^{-1}$, In plane vibration of $s$ - 
triazine ring at $1474 \mathrm{~cm}^{-1}, \mathrm{C}-\mathrm{C}(=\mathrm{O})-\mathrm{O}$ ester stretching vibration at $1752 \mathrm{~cm}^{-1}, \mathrm{C}-\mathrm{H}$ stretching vibration of methyl group at $2780 \mathrm{~cm}^{-1}, \mathrm{C}-\mathrm{H}$ stretching vibration of methylene group $3026 \mathrm{~cm}^{-1}$.

\section{${ }^{1}$ H NMR SPECTROSCOPY:}

The ${ }^{1} \mathrm{H}-\mathrm{NMR}$ spectra of PE 5 (II) at $\delta$ ppm: $8.74(2 \mathrm{H}, \mathrm{s}, \mathrm{Ar}-\mathrm{CH}-), 8.25(2 \mathrm{H}, \mathrm{d}, \mathrm{Ar}-\mathrm{CH}-), 8.00(2 \mathrm{H}, \mathrm{d}, \mathrm{Ar}-\mathrm{CH}-)$, 7. $91(2 \mathrm{H}, \mathrm{d}, \mathrm{Ar}-\mathrm{CH}-), 7.55(2 \mathrm{H}, \mathrm{s}, \mathrm{Ar}-\mathrm{CH}-), 7.22-7.38(11 \mathrm{H}, \mathrm{m}, \mathrm{Ar}-\mathrm{CH}-), 7.00$ (1H, d, Ar-CH-), 6.85 (2H, d, $\mathrm{Ar}$ - $\mathrm{CH}-), 3.69\left(4 \mathrm{H}, \mathrm{t}\right.$, Methylene - $\left.\mathrm{CH}_{2}-\right), 2.14-1.93\left(4 \mathrm{H}, \mathrm{m}\right.$, Methylene $\left.-\mathrm{CH}_{2^{-}}\right), 1.49-1.65\left(12 \mathrm{H}, \mathrm{m}\right.$, methylene $\left.-\mathrm{CH}_{2^{-}}\right)$.

The ${ }^{1} \mathrm{H}$-NMR spectra of PE 8 (II) at $\delta$ ppm: $8.75\left(2 \mathrm{H}, \mathrm{s}, \mathrm{Ar}-\mathrm{CH}_{2^{-}}\right), 8.26\left(2 \mathrm{H}, \mathrm{d}, \mathrm{Ar}-\mathrm{CH}_{2^{-}}\right), 8.00\left(2 \mathrm{H}, \mathrm{d}, \mathrm{Ar}-\mathrm{CH}_{2^{-}}\right)$, $7.93\left(2 \mathrm{H}, \mathrm{d}, \mathrm{Ar}-\mathrm{CH}_{2^{-}}\right), 7.54\left(2 \mathrm{H}, \mathrm{s}, \mathrm{Ar}-\mathrm{CH}_{2^{-}}\right), 7.38\left(1 \mathrm{H}, \mathrm{d}, \mathrm{Ar}-\mathrm{CH}_{2^{-}}\right), 7.10-7.35\left(9 \mathrm{H}, \mathrm{m}, \mathrm{Ar}-\mathrm{CH}_{2^{-}}\right), 3.70(4 \mathrm{H}, \mathrm{t}$, Methylene $-\mathrm{CH}_{2}-$ ), $1.52-1.65\left(6 \mathrm{H}, \mathrm{m}\right.$, Methylene- $\mathrm{CH}_{2}-$ ).

\section{Solubility:-}

Polymer samples that swelled without dissolving at $25{ }^{\circ} \mathrm{C}$ were heated to $50{ }^{\circ} \mathrm{C}$ in order to effect the process of dissolution. Several solvents reported in Table 1 were tested for this purpose.

Table 1:- Solubility of polyesters in different solvents

\begin{tabular}{|c|c|c|c|c|c|c|c|c|c|c|}
\hline Solvent & $\begin{array}{c}\text { PE } 1 \\
\text { (II) }\end{array}$ & $\begin{array}{c}\text { PE } 2 \\
\text { (II) }\end{array}$ & $\begin{array}{c}\text { PE } 3 \\
\text { (II) }\end{array}$ & $\begin{array}{l}\text { PE } 4 \\
\text { (II) }\end{array}$ & $\begin{array}{c}\text { PE } 5 \\
\text { (II) }\end{array}$ & $\begin{array}{c}\text { PE 6 } \\
\text { (II) }\end{array}$ & $\begin{array}{c}\text { PE } 7 \\
\text { (II) }\end{array}$ & $\begin{array}{c}\text { PE 8 } \\
\text { (II) }\end{array}$ & $\begin{array}{c}\text { PE } 9 \\
\text { (II) }\end{array}$ & $\begin{array}{l}\text { PE 10 } \\
\text { (II) }\end{array}$ \\
\hline Chloro Benzene & -- & -- & - & - & -- & -- & - & -- & -- & -- \\
\hline Benzene & -- & -- & - & -- & -- & - & - & -- & -- & - \\
\hline Toluene & -- & - & - & -- & -- & -- & - & -- & -- & -- \\
\hline Chloroform & -- & -- & -- & -- & -- & -- & -- & - & -- & -- \\
\hline Acetone & ++ & ++ & ++ & ++ & ++ & ++ & ++ & ++ & ++ & ++ \\
\hline $\begin{array}{l}\text { Carbon } \\
\text { Tetrachloride }\end{array}$ & -- & - & -- & - & - & - & - & - & - & -- \\
\hline $\begin{array}{l}\text { Di Methyl } \\
\text { Formamide }\end{array}$ & ++ & ++ & ++ & ++ & ++ & ++ & ++ & ++ & ++ & ++ \\
\hline Ethyl Acetate & ++ & ++ & ++ & ++ & ++ & ++ & ++ & ++ & ++ & ++ \\
\hline Methanol & -- & -- & -- & -- & -- & -- & -- & - & -- & -- \\
\hline Ethanol & -- & - & - & -- & -- & - & - & - & -- & -- \\
\hline DMSO & ++ & ++ & ++ & ++ & ++ & ++ & ++ & ++ & ++ & ++ \\
\hline 1,4-Dioxane & ++ & ++ & ++ & ++ & ++ & ++ & ++ & ++ & ++ & ++ \\
\hline $\begin{array}{ll}1,2 & \text { Dichloro } \\
\text { Ethane } & \\
\end{array}$ & \pm+ & \pm+ & \pm+ & \pm+ & \pm+ & \pm+ & \pm+ & \pm+ & \pm+ & \pm+ \\
\hline $\begin{array}{ll}2 & \text { Chloro } \\
\text { Propanol } & \\
\end{array}$ & \pm+ & \pm+ & \pm+ & \pm+ & \pm+ & \pm+ & \pm+ & \pm+ & \pm+ & \pm+ \\
\hline Pyridine & ++ & ++ & ++ & ++ & ++ & ++ & ++ & ++ & ++ & ++ \\
\hline
\end{tabular}

$+=$ Soluble, $-=$ Insoluble, $+=$ Partlysoluble, $1^{\text {st }}$ symbole indicates $25+1{ }^{\circ} \mathrm{C} 2^{\text {nd }}$ at $50+1{ }^{\circ} \mathrm{C}$.

Density characteristics:-

The density of each copolyster were determine at $25 \pm 3{ }^{\circ} \mathrm{C}$ by suspending each of the polyesters in a liquid mixture of carbon tetrachloride and acetone and subsequently measuring the density of the liquid mixture by a pycnometer, are presented in Table 2. Density of all the copolyesters varies from 1.204 to $1.165 \mathrm{~g} / \mathrm{cm}^{3}$. The density of copolyesters varies with the different diols used, and decreased in the following order:

PE 2 (II) $>$ PE 7 (II) $>$ PE 6 (II) $>$ PE 8 (II) $>$ PE 9 (II) $>$ PE 1 (II) $>$

$$
\text { PE } 3 \text { (II) }>\text { PE } 5 \text { (II) }>\text { PE } 10 \text { (II) }>\text { PE } 4 \text { (II). }
$$

Table 2:- Density of Copolyesters

\begin{tabular}{|l|l|l|l|}
\hline Polymer Code & Yield (\%) & Colour & Density $\left(\mathbf{g} / \mathbf{c m}^{\mathbf{3}}\right)$ \\
\hline PE 1 (II) & 71 & Brown & 1.187 \\
\hline PE 2 (II) & 75 & Brown & 1.204 \\
\hline PE 3 (II) & 83 & Brown & 1.175 \\
\hline PE 4 (II) & 68 & Brown & 1.165 \\
\hline PE 5 (II) & 84 & Brown & 1.175 \\
\hline PE 6 (II) & 79 & Black-Brown & 1.195 \\
\hline
\end{tabular}




\begin{tabular}{|l|l|l|l|}
\hline PE 7 (II) & 80 & Brown & 1.199 \\
\hline PE 8 (II) & 71 & Brown & 1.193 \\
\hline PE 9 (II) & 67 & Brown & 1.192 \\
\hline PE 10 (II) & 73 & Brown & 1.169 \\
\hline
\end{tabular}

Viscosity Measurements:-

Intrinsic viscosity, reduced viscosity and inherent viscosity for all the polyesters at various concentration were determined at $25 \pm 1{ }^{\circ} \mathrm{C}$. Typical Huggins \& Kraemer plots were used to obtain intrinsic viscosity for each of polyesters. Solution viscosity of PE 1 (II) at different concentration is shown in Table 3. The Intrinsic viscosity of the polyesters synthesized from PNCCT and various mixture of diols, decreasing in the following sequence given below:

PE 6 (II) $>$ PE 8 (II) $>$ PE 3 (II) > PE 1 (II) $>$ PE 4 (II) > PE 7 (II) $>$ PE 2 (II) $>$ PE 10 (II) $>$ PE 9 (II) $>$ PE 5 (II)

Table 3:- Solution viscosity of copolyester PE 1 (II) Solvent: DMF $t_{0}=172$ seconds

\begin{tabular}{|c|l|l|l|l|l|l|}
\hline Sr. No. & Con. C (g/dl) & $\begin{array}{l}\text { Flow time } \\
(\mathbf{t} \mathbf{~ s e c})\end{array}$ & $\eta_{\text {rel }}=\mathbf{t} / \mathbf{t}_{\mathbf{0}}$ & $\eta_{\text {sp. }=} \eta_{\text {rel- }} \mathbf{1}$ & $\eta_{\text {red }}=\eta_{\text {sp }} / \mathbf{C}$ & $\ln \eta_{\text {rel }} / \mathbf{C}$ \\
\hline $\mathbf{1 .}$ & 0.2 & 187.2 & 1.0883 & 0.0883 & 0.4415 & 0.423 \\
\hline $\mathbf{2 .}$ & 0.4 & 202.7 & 1.1784 & 0.1784 & 0.446 & 0.410 \\
\hline $\mathbf{3 .}$ & 0.6 & 218.8 & 1.2721 & 0.2721 & 0.453 & 0.401 \\
\hline $\mathbf{4 .}$ & 0.8 & 235.3 & 1.3680 & 0.3680 & 0.460 & 0.392 \\
\hline $\mathbf{5 .}$ & 1.0 & 252.2 & 1.4662 & 0.4662 & 0.466 & 0.382 \\
\hline
\end{tabular}

Huggins \& Kraemer constant for all polyesters were derived from the graph (Figures 1 to 5 ) is shown in the Table 4.


Figure_4 Huggins \& Kraemer plot for intrinsic viscosity of PE 1 (II) \& PE 2(II)
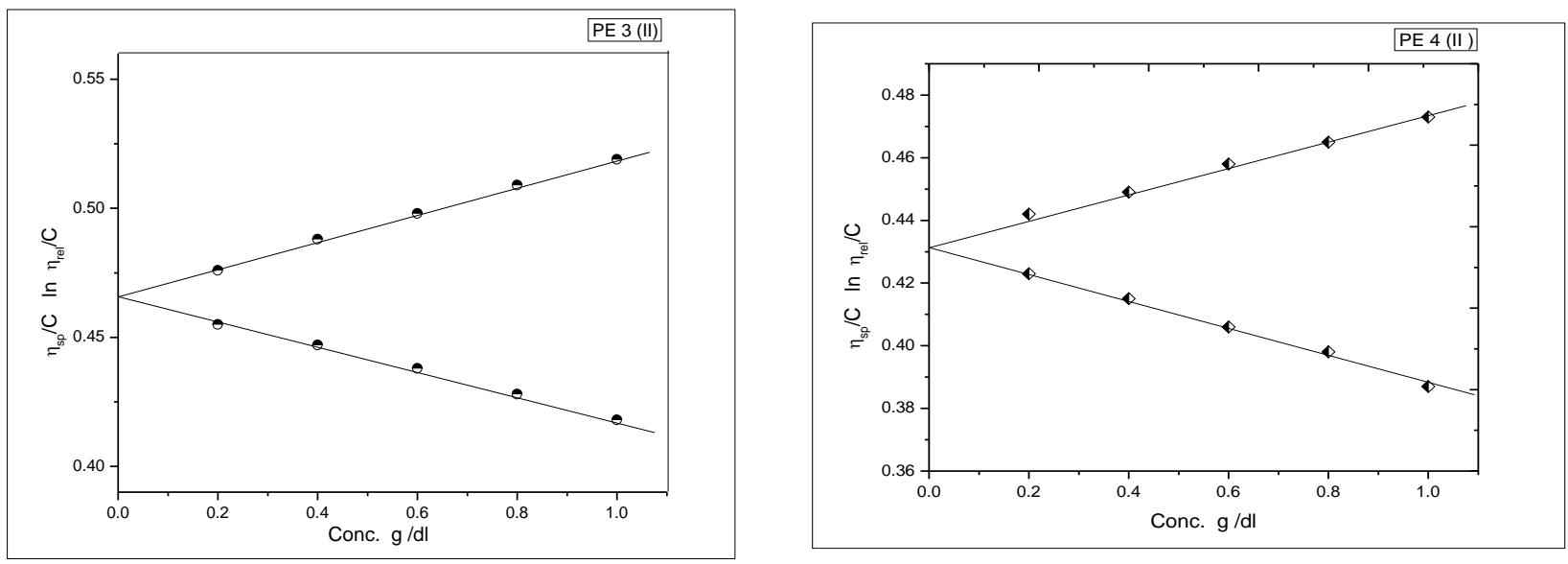
Figure_5 Huggins \& Kraemer plot for intrinsic viscosity of PE 3 (II) \& PE 4 (II)
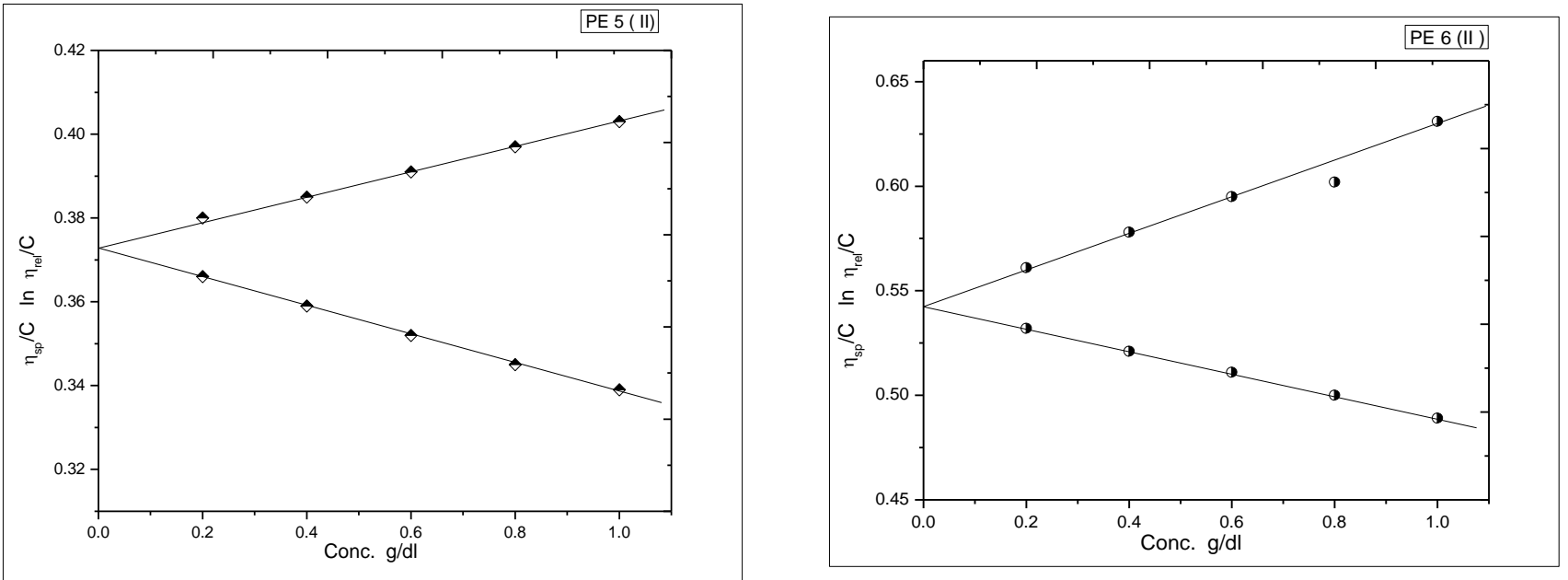

Figure_6 Huggins \& Kraemer plot for intrinsic viscosity of PE 5 (II) \& PE 6 (II)
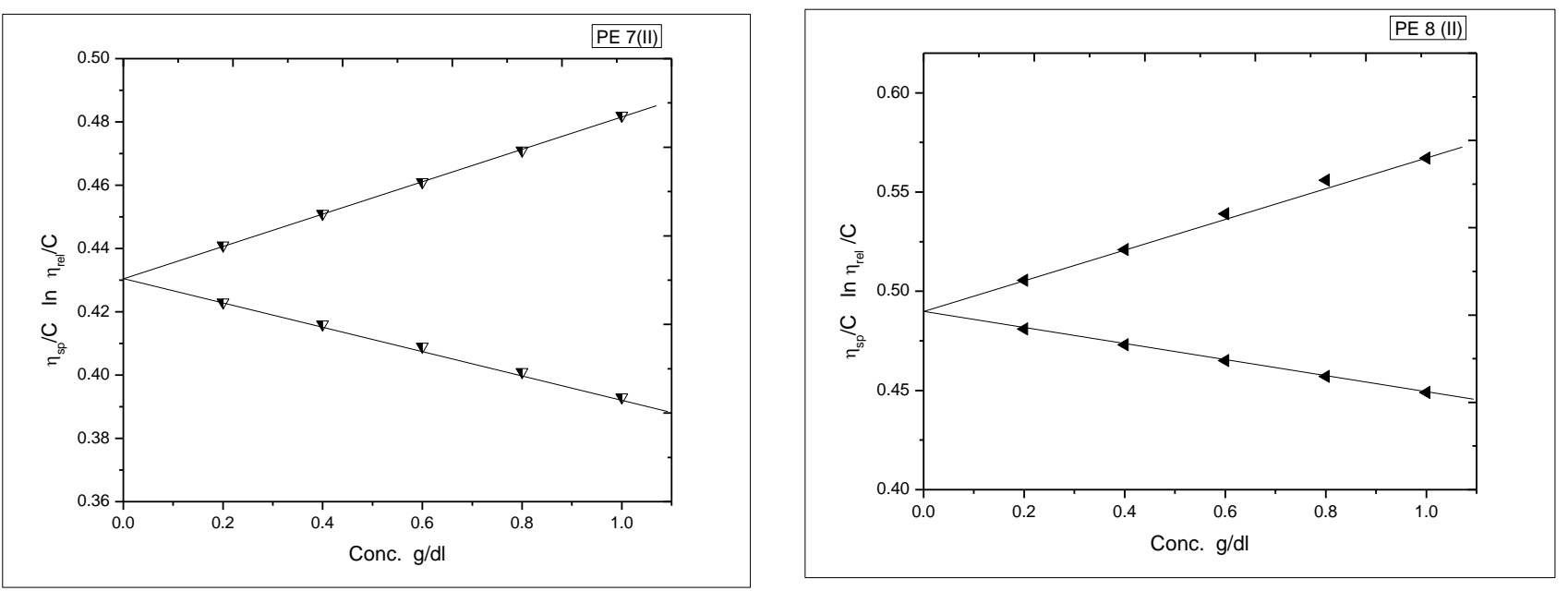

Figure_7 Huggins \& Kraemer plot for intrinsic viscosity of PE 7 (II) \& PE 8 (II)
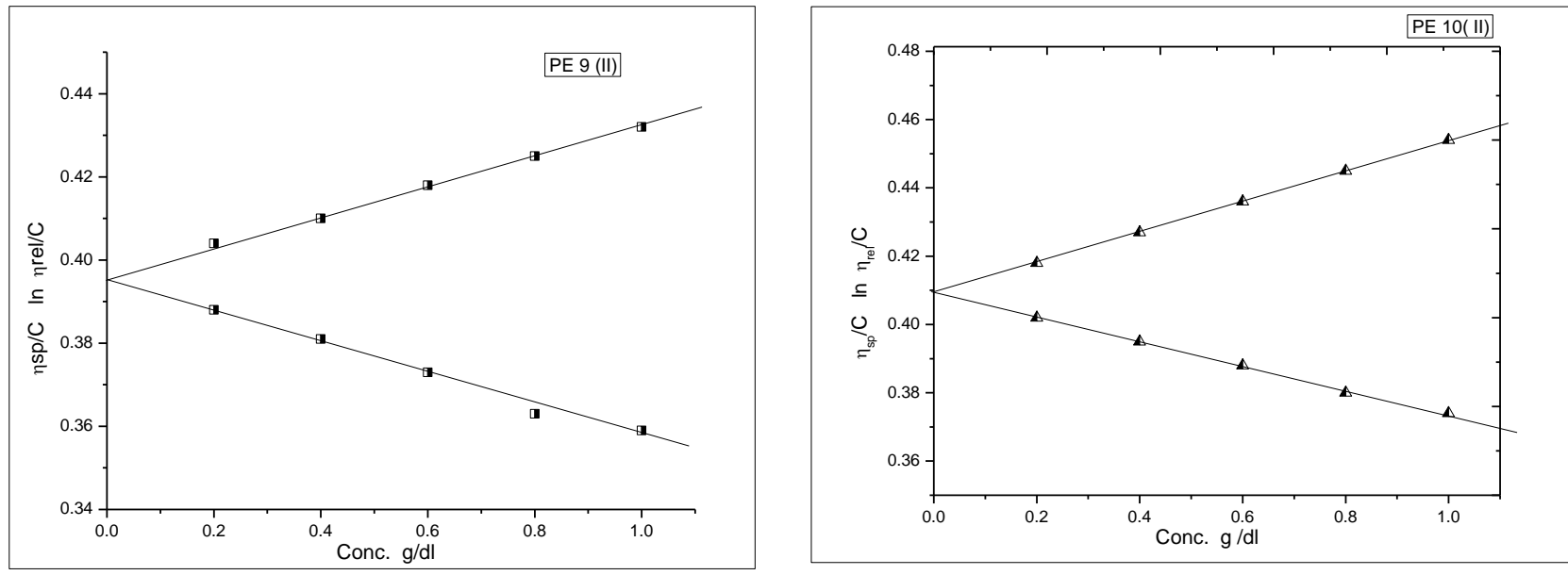

Figure_8 Huggins \& Kraemer plot for intrinsic viscosity of PE 9 (II) \& PE 10 (II)

Table 4:- Viscosity values and Huggins \& Kraemer constant for copolyesters:

\begin{tabular}{|l|l|l|l|l|l|l|l|}
\hline Polymer Code & Intrinsic & Reduced & Inherent & Huggins & Kraemer & Huggins & Kraemer \\
\hline
\end{tabular}




\begin{tabular}{|l|l|l|l|l|l|l|l|}
\hline & $\begin{array}{l}\text { Viscosity } \\
{[\eta]}\end{array}$ & $\begin{array}{l}\text { Viscosity } \\
\eta_{\text {sp }} / \mathbf{C}\end{array}$ & $\begin{array}{l}\text { Viscosity } \\
\mathbf{l n} \eta_{\text {rel }} / \mathbf{C}\end{array}$ & slope & slope & $\begin{array}{l}\text { Constant } \\
\text { K }\end{array}$ & $\begin{array}{l}\text { Constant } \\
\boldsymbol{\beta}\end{array}$ \\
\hline PE 1 (II) & 0.434 & 0.466 & 0.382 & 0.0325 & 0.052 & 0.171 & 0.278 \\
\hline PE 2 (II) & 0.415 & 0.475 & 0.388 & 0.060 & 0.027 & 0.348 & 0.156 \\
\hline PE 3 (II) & 0.466 & 0.519 & 0.418 & 0.057 & 0.042 & 0.244 & 0.221 \\
\hline PE 4 (II) & 0.431 & 0.473 & 0.387 & 0.050 & 0.043 & 0.227 & 0.237 \\
\hline PE 5 (II) & 0.373 & 0.403 & 0.338 & 0.027 & 0.033 & 0.215 & 0.251 \\
\hline PE 6 (II) & 0.543 & 0.630 & 0.489 & 0.085 & 0.055 & 0.295 & 0.183 \\
\hline PE 7 (II) & 0.431 & 0.481 & 0.393 & 0.055 & 0.045 & 0.270 & 0.205 \\
\hline PE 8 (II) & 0.490 & 0.567 & 0.449 & 0.080 & 0.050 & 0.320 & 0.170 \\
\hline PE 9 (II) & 0.395 & 0.431 & 0.359 & 0.040 & 0.033 & 0.230 & 0.230 \\
\hline PE 10 (II) & 0.409 & 0.454 & 0.374 & 0.050 & 0.036 & 0.269 & 0.209 \\
\hline
\end{tabular}

Thermo Gravimetric Analysis:-

TGA of PE 8 (II) and PE 9 (II) obtained at a heating rate of $10{ }^{\circ} \mathrm{C} / \mathrm{min}$. in nitrogen atmosphere showed in Figures 9 $\& 10$. The characteristic data are presented in Table 5 gave the following information.

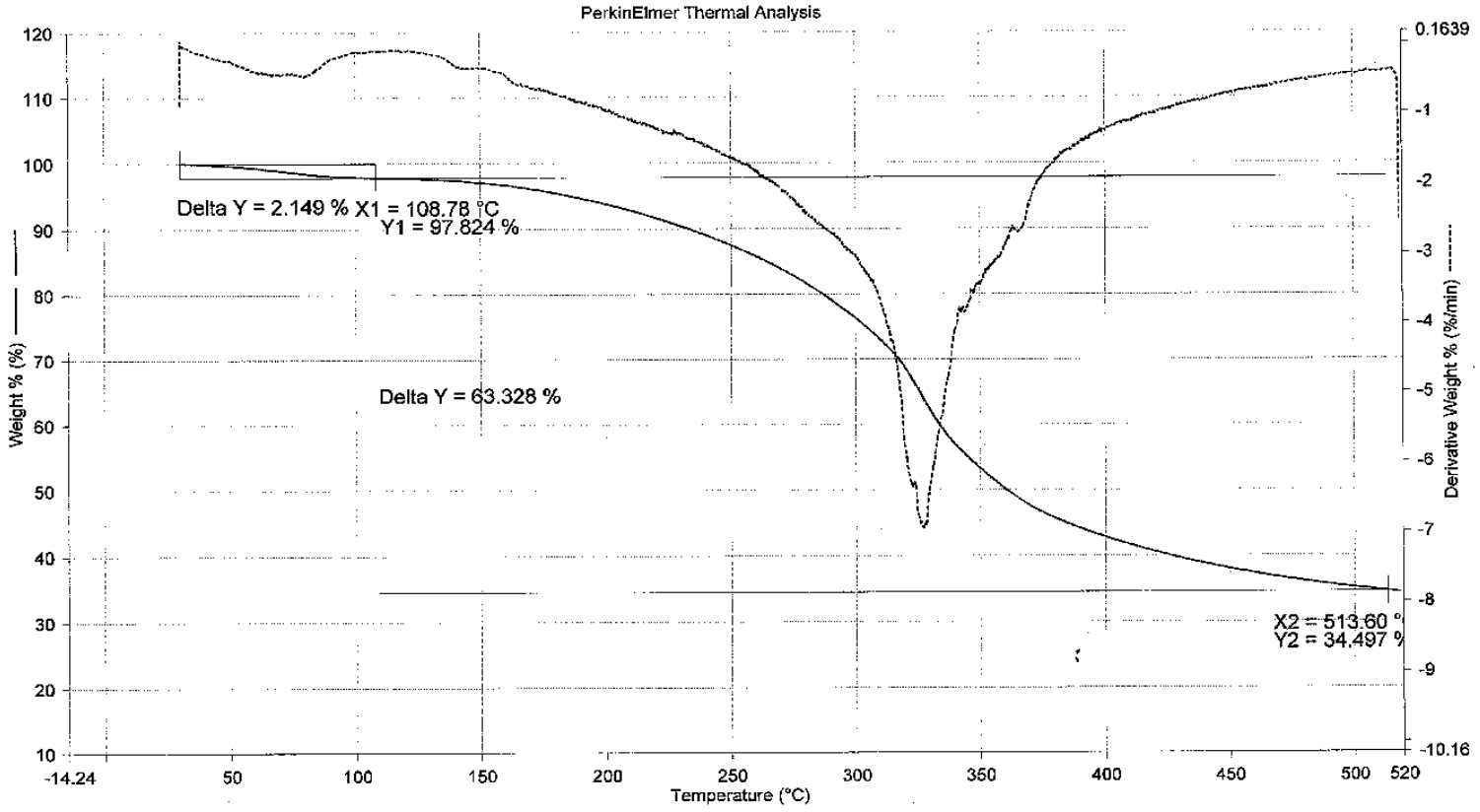

Figure_9 TGA of PE 8 (II) 


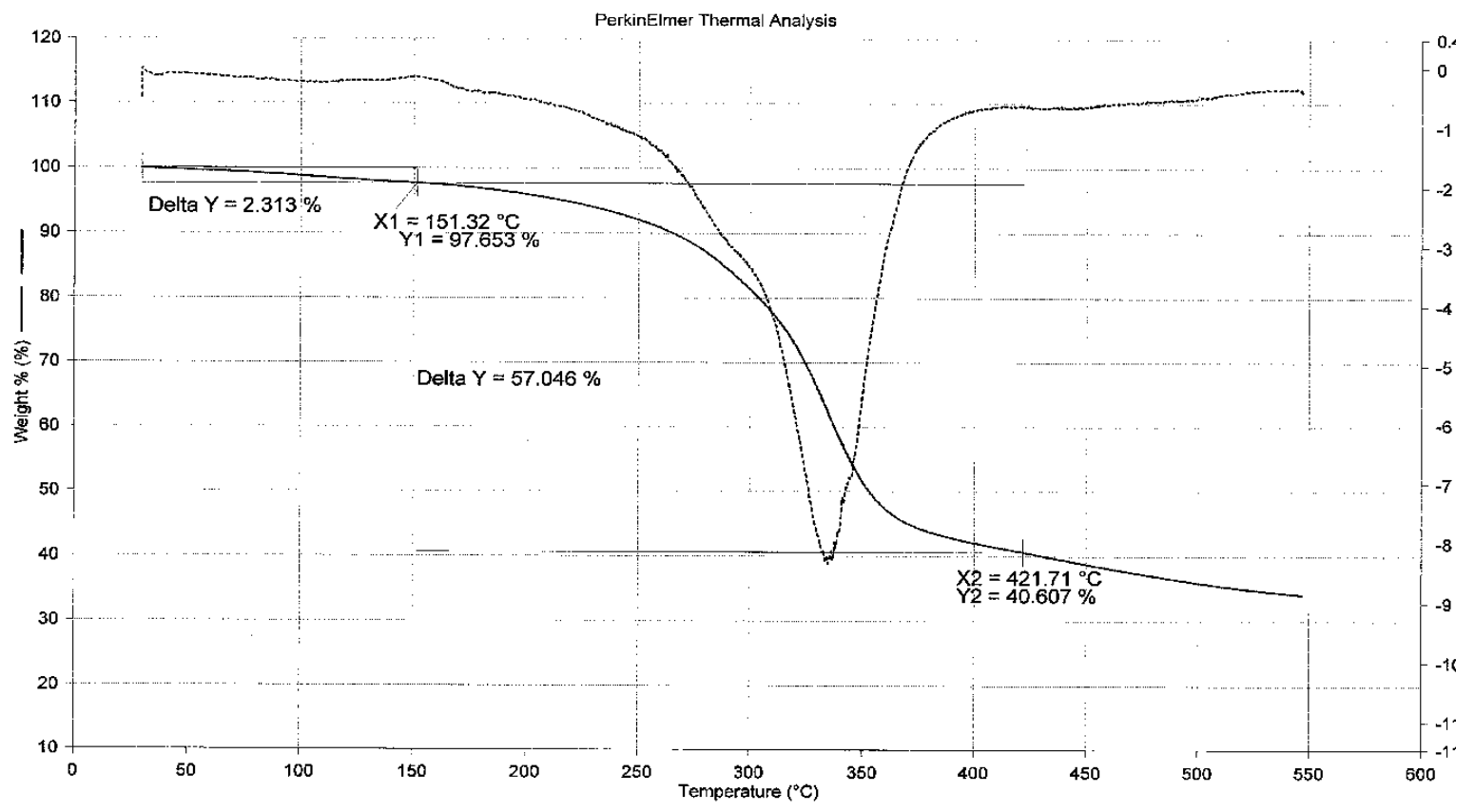

Figure_10 TGA of PE 9 (II)

Table 5:- Thermal data of PE 8 (II) \& PE 9 (II)

\begin{tabular}{|l|l|l|l|l|l|}
\hline Polymer & $\mathbf{T}_{\mathbf{0}}$ & $\mathbf{T}_{\mathbf{1 0}}$ & $\mathbf{T}_{\max }$ & \multirow{2}{*}{$\mathbf{T}_{\mathbf{S}}$} \\
\cline { 1 - 4 } & & & Step I & Step II & \\
\hline PE 8 (II) & 140 & 240 & 320 & - & 335 \\
\hline PE 9 (II) & 170 & 260 & 330 & - & 350 \\
\hline
\end{tabular}

$\mathrm{T}_{0}=$ Initial decomposition temperature.

$\mathrm{T}_{10}=$ Temperature for $10 \%$ weight loss.

$\mathrm{T}_{\max }=$ Temperature for maximum rate of decomposition.

$\mathrm{T}_{\mathrm{S}}=$ Half volatisation temperature.

PE 8 (II) decomposes single distinct weight loss step. The polymer begins to decompose at about $150{ }^{\circ} \mathrm{C}$. The decomposition is marked with a rapid weight loss in the temperature range $200-450{ }^{\circ} \mathrm{C}$ during which the polymer samples losses $54 \%$ of its weight. The maximum rate of weight loss occurs at $320{ }^{\circ} \mathrm{C}$. After $450{ }^{\circ} \mathrm{C}$ the samples decomposes up to $520{ }^{\circ} \mathrm{C}$ leaving about $34 \%$ residue.

PE 9 (II) decomposes single distinct weight loss step. The polymer begins to decompose at about $170{ }^{\circ} \mathrm{C}$. The decomposition is marked with a rapid weight loss in the temperature range $190-400{ }^{\circ} \mathrm{C}$ during which the polymer samples losses $54 \%$ of its weight. The maximum rate of weight loss occurs at $330{ }^{\circ} \mathrm{C}$. After $450{ }^{\circ} \mathrm{C}$ the samples decomposes up to $550{ }^{\circ} \mathrm{C}$ leaving about $34 \%$ residue.

The thermal stability of copolyester on the basis of initial decomposition temperature $\mathrm{T}_{0}$ is found as follow:

PE 9 (II) > PE 8 (II).

Thermograms have been analyzed as per the graphical methods, proposed by Broido and Horowitz \& Metzger.

Broido and Horowitz \& Metzger methods are exemplified for PE 8 (II) in Table 6 \& 7 respectively while for PE 9 (II) is shown in Tables $8 \& 9$ and the corresponding plots are shown in Figures $6 \& 7$ and $8 \& 9$.

Table 6:- Application of Broido method to thermogram of PE 8 (II):

\begin{tabular}{|l|l|l|l|l|l|l|}
\hline $\mathbf{T}^{\circ} \mathbf{C}$ & \% Wt. & $\mathbf{Y}=\frac{\mathbf{W}_{\mathbf{t}}-\mathbf{W} \infty}{\mathbf{W}_{\mathbf{0}}-\mathbf{W} \infty}$ & $\mathbf{1} / \mathbf{Y}$ & $\mathbf{l n} \mathbf{I n}(\mathbf{1} / \mathbf{Y})$ & $\mathbf{T ~ K}$ & $\begin{array}{l}\mathbf{1 0 0 0 / \mathbf { T }} \\
\left(\mathbf{K}^{-\mathbf{1}}\right)\end{array}$ \\
\hline $\mathbf{2 9 0}$ & 79.42 & 0.6858 & 1.4581 & -0.9751 & 563 & 1.7761 \\
\hline $\mathbf{3 0 0}$ & 76.80 & 0.6458 & 1.5484 & -0.8273 & 573 & 1.745 \\
\hline
\end{tabular}




\begin{tabular}{|l|l|l|l|l|l|l|}
\hline 310 & 75.20 & 0.6212 & 1.6097 & -0.7422 & 583 & 1.715 \\
\hline 320 & 70.15 & 0.5443 & 1.8372 & -0.1971 & 593 & 1.686 \\
\hline $\mathbf{3 3 0}$ & 62.74 & 0.4312 & 2.3191 & -0.1729 & 603 & 1.658 \\
\hline $\mathbf{3 4 0}$ & 56.92 & 0.3424 & 2.9205 & 0.0692 & 613 & 1.631 \\
\hline $\mathbf{3 5 0}$ & 53.46 & 0.2895 & 3.4542 & 0.447 & 623 & 1.605 \\
\hline
\end{tabular}

Table 7:- Application of Horowitz- Metzger method to thermogram of PE 8 (II):

\begin{tabular}{|l|l|l|l|l|l|l|}
\hline $\mathbf{T}^{\circ} \mathbf{C}$ & \% Wt. & $\mathbf{1}-\boldsymbol{\alpha}$ & $\mathbf{1 / 1}-\boldsymbol{\alpha}$ & $\ln \ln (\mathbf{1} / \mathbf{1}-\boldsymbol{\alpha})$ & $\mathbf{T ~ K}$ & $\boldsymbol{\Theta}$ \\
\hline $\mathbf{3 8 0}$ & 380 & 79.42 & 0.6858 & 1.4581 & -0.9751 & -30 \\
\hline $\mathbf{3 9 0}$ & 390 & 76.80 & 0.6458 & 1.5484 & -0.8273 & -20 \\
\hline $\mathbf{4 0 0}$ & 400 & 75.20 & 0.6212 & 1.6097 & -0.7422 & -10 \\
\hline $\mathbf{4 1 0}$ & 410 & 70.15 & 0.5443 & 1.8372 & -0.1971 & 00 \\
\hline $\mathbf{4 2 0}$ & 420 & 62.74 & 0.4312 & 2.3191 & -0.1729 & 10 \\
\hline $\mathbf{4 3 0}$ & 430 & 56.92 & 0.3424 & 2.9205 & 0.0692 & 20 \\
\hline $\mathbf{4 4 0}$ & 440 & 53.46 & 0.2895 & 3.4542 & 0.447 & 30 \\
\hline
\end{tabular}

Table 8:- Application of Broido method to thermogram of PE 9 (II):

\begin{tabular}{|c|c|c|c|c|c|c|}
\hline $\mathbf{T}^{\circ} \mathbf{C}$ & $\% \mathrm{Wt}$. & $\mathbf{Y}=\frac{\mathbf{W}_{\mathrm{t}}-\mathbf{W}_{\infty}}{\mathbf{W}_{\mathbf{0}}-\mathbf{W}_{\infty}}$ & $1 / \mathrm{Y}$ & $\ln \ln (1 / Y)$ & T K & $\begin{array}{c}1000 / T \\
\left(K^{-1}\right)\end{array}$ \\
\hline 300 & 81.48 & 0.6282 & 1.5918 & -0.7659 & 573 & 1.745 \\
\hline 310 & 77.87 & 0.5558 & 1.7992 & -0.5321 & 583 & 1.715 \\
\hline 320 & 73.08 & 0.4596 & 2.1758 & -0.2518 & 593 & 1.686 \\
\hline 330 & 66.36 & 0.4336 & 2.3062 & -0.1795 & 603 & 1.658 \\
\hline 340 & 58.30 & 0.2979 & 3.3568 & 0.1914 & 613 & 1.631 \\
\hline 350 & 51.60 & 0.1851 & 5.4024 & 0.5228 & 623 & 1.605 \\
\hline 360 & 47.42 & 0.1148 & 8.7108 & 0.7722 & 633 & 1.579 \\
\hline
\end{tabular}

Table 9:- Application of Horowitz- Metzger method to thermogram of PE 9 (II):

\begin{tabular}{|l|l|l|l|l|l|l|}
\hline $\mathbf{T}^{\circ} \mathbf{C}$ & \% Wt. & $\mathbf{1 - \alpha}$ & $\mathbf{1 / 1} \boldsymbol{\alpha}$ & $\ln \ln (\mathbf{1} / \mathbf{1}-\boldsymbol{\alpha})$ & $\mathbf{T ~ K}$ & $\boldsymbol{\Theta}$ \\
\hline $\mathbf{3 0 0}$ & 81.48 & 0.6282 & 1.5918 & -0.7659 & 573 & -30 \\
\hline $\mathbf{3 1 0}$ & 77.87 & 0.5558 & 1.7992 & -0.5321 & 583 & -20 \\
\hline $\mathbf{3 2 0}$ & 73.08 & 0.4596 & 2.1758 & -0.2518 & 593 & -10 \\
\hline $\mathbf{3 3 0}$ & 66.36 & 0.4336 & 2.3062 & -0.1795 & 603 & 00 \\
\hline $\mathbf{3 4 0}$ & 58.30 & 0.2979 & 3.3568 & 0.1914 & 613 & 10 \\
\hline $\mathbf{3 5 0}$ & 51.60 & 0.1851 & 5.4024 & 0.5228 & 623 & 20 \\
\hline $\mathbf{3 6 0}$ & 47.42 & 0.1148 & 8.7108 & 0.7722 & 633 & 30 \\
\hline
\end{tabular}






Figure_11 Broido plot for PE 8 (II)

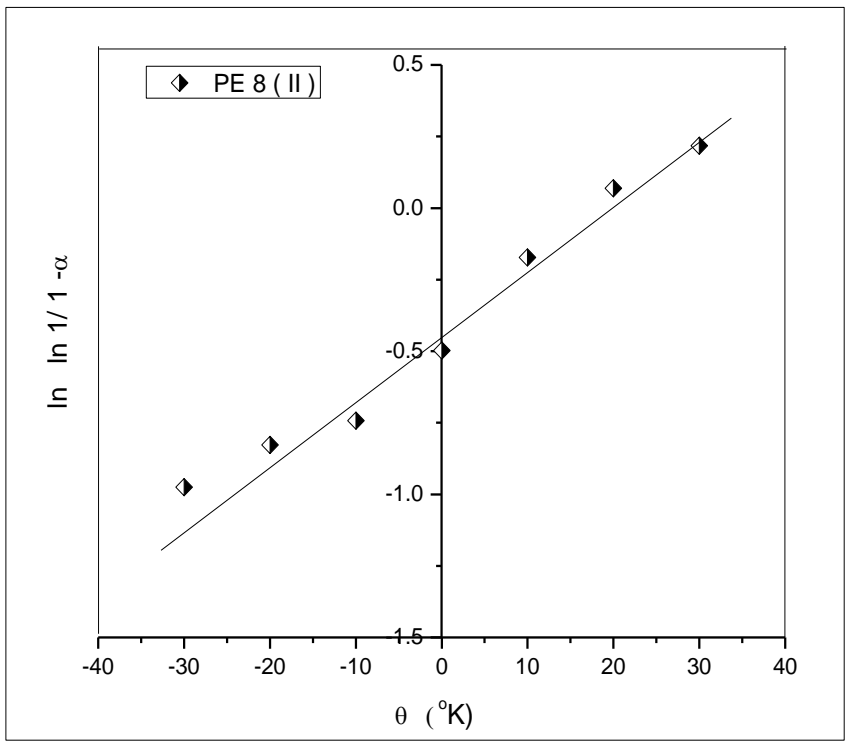

Figure _12 Horowitz- Metzger plot for PE 8 (II) 


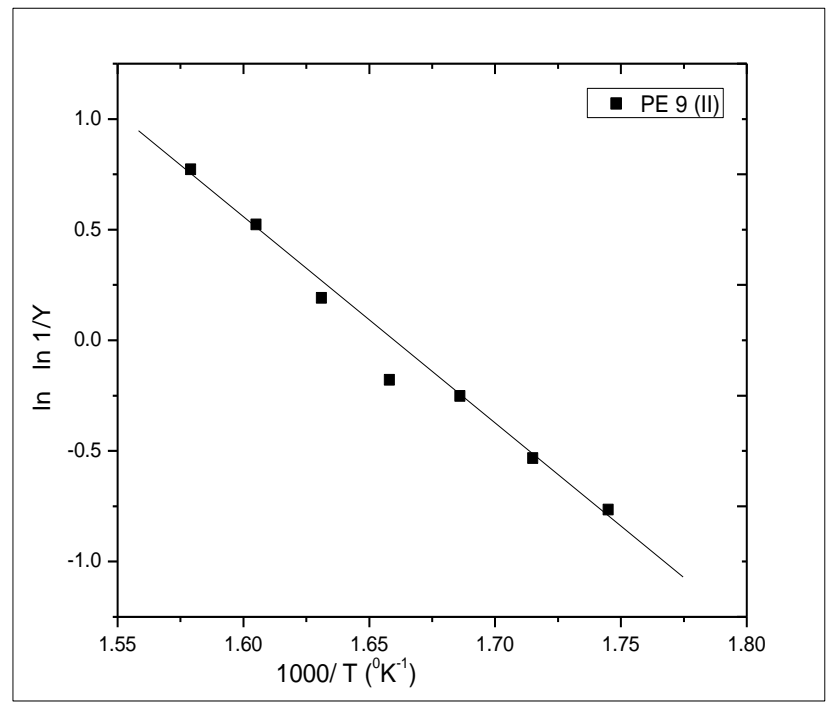

Figure_13 Broido plot for PE 9 (II)

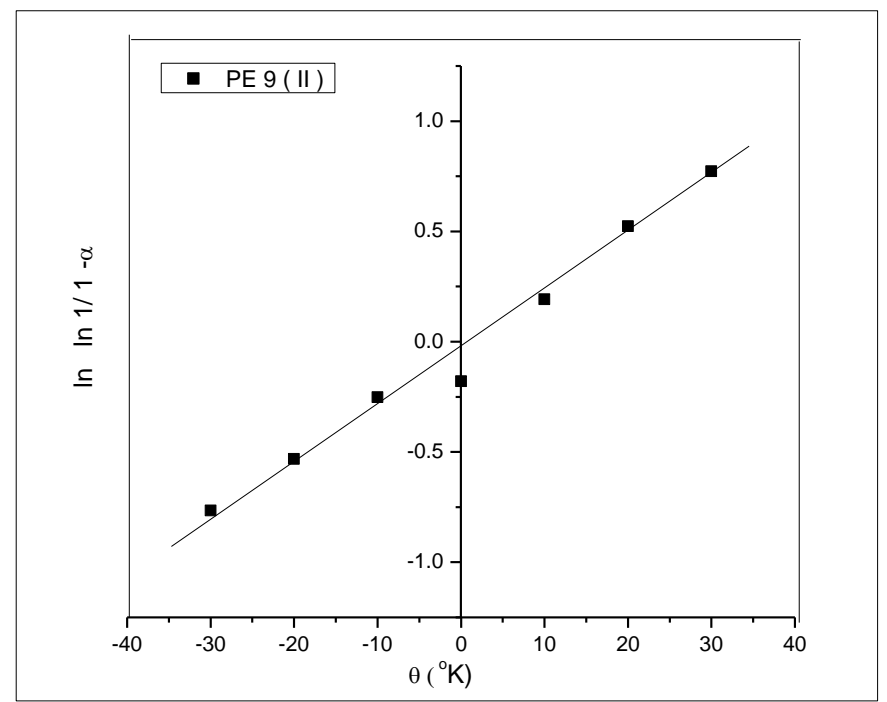

Figure_14 Horowitz Metzger plot for PE 9 (II)

Activation energy calculated by Broido and Horowitz \& Metzger method which given in the Table 10.

Table 10:- Activation energy calculated by Broido and Horowitz \& Metzger method:

\begin{tabular}{|l|l|l|l|l|l|}
\hline \multirow{2}{*}{ Polymer code } & \multicolumn{4}{|l|}{ Broido } & \multicolumn{4}{l|}{ Horowitz \& Metzger } \\
\cline { 2 - 6 } & Slope & Ea $(\mathbf{K . C a l} / \mathbf{m o l})$ & Slope & Ts & Ea $(\mathbf{K . C a l} / \mathbf{m o l})$ \\
\hline PE 8 (II) & -10.0 & 19.87 & 0.029 & 608 & 21.30 \\
\hline PE 9 (II) & -9.50 & 18.91 & 0.018 & 623 & 18.69 \\
\hline
\end{tabular}

\section{Conclusion:-}

A series of copolyesters were synthesized from diacid chloride of 2-( $N$-piperidino)-4, 6-dichloro-s-triazine [PDCT] and various mixtures of diols. The synthesized polyesters have been found to be soluble in some common solvents like acetone, DMF and DMSO. Density of all the copolyesters varies from 1.204 to $1.165 \mathrm{~g} / \mathrm{cm}^{3}$. The intrinsic viscosity, reduced viscosity and inherent viscosity were determined by using Huggins \& Kraemer method. The activation energy was calculated by Broido and Horowitz \& Metzger method for PE 8 (II) and PE 9 (II) were found 
21.30 and $18.69 \mathrm{kcal} / \mathrm{mol}$ respectively. The synthesized polyesters were found to be stable more than $300{ }^{\circ} \mathrm{C}$ temperature, thus the polymers can be used for the high temperature application.

\section{Acknowledgements:-}

Author like to thank the department of chemistry for providing facilities for research. One of the author also like to thank UGC-BSR for financial support.

Symbols and abbreviations:-

Hq: Hydroquinone,

C: Catachole,

BPC: Bisphenol-C,

BPS: Bisphenol-S

PE 1 (II): PE (Hq + BPA)

PE 2 (II): PE (C + BPA)

PE 3 (II): $\mathrm{PE}(\mathrm{R}+\mathrm{BPA})$

PE 4 (II): PE (C + BPC)

PE 5 (II): PE (R + BPC)
BPA: Bisphenol-A,

R: Resorcinol,

Ph: Phenolphthalenine,

PE: Polyester,

PE 6 (II): PE (BPC + BPS)

PE 7 (II): $\mathrm{PE}(\mathrm{Hq}+\mathrm{Ph})$

PE 8 (II): $\mathrm{PE}(\mathrm{Hq}+\mathrm{C})$

PE 9 (II): $\mathrm{PE}(\mathrm{Hq}+\mathrm{BPS})$

PE 10 (II): PE (Hq + EG)

\section{Reference:-}

1. J. P. Critchley, G. J. Knight and W. W. Wright Heat-resistant polymers, Plenum Press, New York, (1983).

2. R. B. Seymour and G. S. Krishenbaum, "High Performance Polymers: Their Origin and Development," Elsevier, New York, (1986).

3. S. W. Kim, S. C. Shim, B. J. Jung and H. K. Shim, Synthesis and properties of new Polymer, J. Polym., Elseviers, 43, 4297 (2002).

4. D. M. Lewis and B. Voncina, Durable press finishing of cotton with polycarboxylic acids and preparation of thiosuccinyl-s-triazine, J. Appl. Polym. Sci., 81, 124 (2001).

5. W. T. Wang and S.C. Wu The liquid crystalline state of polyimide precursors. J. Polym. Sci., Polym. Chem. Ed., 26, 2749 (1988).

6. KUS Canan, Synthesis of new substituted 6-(morpholin4-yl)-1H-benzimidazole derivatives, Turk. J. Chem., 26, 559, (2002).

7. P. N. Sojitra, K. C. Patel and H. S. Patel, High Performance Polyamides based on s-Triazine Ring: Synthesis and Characterization, High Performance polymers, 22, 974-988 (2010).

8. P. N. Sojitra and K. C. Patel, Synthesis of polyamides by energy-efficient ultrasonic-assisted direct polycondensation, High Performance polymers, 23(8), 565-574 (2011).

9. H. S. Patel, V.C. Patel, Polyimides containing s-triazine ring, Eur. Polym. J., 37, 2263-2271, 2001.

10. H. S. Patel and K. C. Patel, Synthesis and Properties of Novel Polycyanurates Derived from 2-( $N$-piperidino)4,6-bis (naphthoxy-2-carbonyl chloride)-s-triazine, , Int. J. Polym. Mater., 55, 1043 (2006).

11. H. S. Patel, R. R. Shah and K. C. Patel, Synthesis and Characterization of Polyamides Based on s-Triazine Moiety, Int. J. Polym. Mater., 56, 499 (2007).

12. H. S. Patel, N. B. Patel and K. C. Patel, Studies on Synthesis and Characterization of some Novel Aromatic Copolyesters based on $s$-Triazine, Iran. Polym. J., 14 (12), 1090-1098, 2005.

13. S. T. Asundaria, H. S. Patel and K. C. Patel, Synthesis and studies of homopolyamides based on 2,4-bis(6chlorocarbonyl-2-naphthyloxy)-6-(4-methyl-1-piperazinyl)-s-triazine, Monatsh Chem, 141:917-927, (2010).

14. P. M. Patel, K. C. Patel and S. K. Patel, Studies on synthesis, viscosity, infrared spectra and thermogravimetric analysis of polyesters containing s-triazine ring in the main chain Malaysian Polymer Journal, Vol. 5, No. 1, p 55-67, (2010).

15. B. V. Patel, H. S. Patel, Copolyesters based on s-triazine moiety: Synthesis and characterization, Indian Journal of Chemistry, 47(B), 885-891, (2008).

16. S. T. Asundaria and K. C. Patel, Synthesis and characterization of novel copolyamides based on $s$-triazine derivatives, Int. J. Polym. Mater., 59, 370-386 (2010).

17. S. T. Asundaria, V. B. Patel and K. C. Patel, Synthesis and study of polycyanurates based on 2-carbazol-4, 6dichloro-s-triazine, Polymer journal, 44 (5), 401-409, (2012).

18. Chun Soo Lee and Kazuyuki Maekawa, Agricultural and Biological Chemistry, 40, 4, 785-790 (2016).

19. S. T. Asundaria, V. B. Patel and K. C. Patel, Synthesis and studies of homopolycyanurates based on 2-carbazol4, 6-dichloro-s-triazine, Russian journal of general chemistry 81 (10), 2183-2192, 2011. 
20. M. L. Huggins, Theory of Solutions of High Polymers, J. Am. Chem. Soc., 64, 2716 (1942).

21. E. O. Kraemer, Molecular Weights of Celluloses and Cellulose Derivates, Ind. Eng. Chem. Anal., 30, 1200 (1938).

23. A. J. Broido, Thermal Analysis, Polym. Sci., A-27, 1761 (1969).

24. H. H. Horowitz and G. Metzger, A New Analysis of Thermogravimetric Traces, Anal. Chem., 35, 1464 (1963). 